Supporting Information for

\title{
Net Contribution of Hydrophobic Association to the Thickening Power of Hydrophobically Modified Polyelectrolytes Prepared by Micellar Polymerization
}

Rui Zhu, ${ }^{\dagger}$ Yujun Feng, ${ }^{*} \dagger$ Pingya Luo ${ }^{\ddagger}$

${ }^{\dagger}$ State Key Laboratory of Polymer Materials Engineering, Polymer Research Institute, Sichuan University, Chengdu 610065, People’s Republic of China

₹ State Key Laboratory of Oil and Gas Exploitation and Development, Southwest Petroleum University, Chengdu 610500, People’s Republic of China

*E-mail: yjfeng@scu.edu.cn 


\section{S1. Materials}

Dodecylpyridinium chloride (98\%, TCI) was used as received.

\section{S2. Recipe Used for Copolymer Synthesis}

The recipes used for the preparation of hydrophobically modified polyelectrolytes (HMPEs) is listed in Table S1. The amounts of stearyl methacrylate (SMA) and sodium dodecyl sulfate (SDS) were adjusted to prepare numerous HMPEs with different hydrophobe contents and lengths of hydrophobic blocks $\left(N_{\mathrm{H}}\right)$.

Table S1. Recipes Used for Copolymer Synthesis

\begin{tabular}{cccccc}
\hline Sample & AA & SMA & SDS & Reaction time & $N_{\mathrm{H}}$ \\
& $(\mathrm{mol} \%)$ & $(\mathrm{mol} \%)$ & $(\mathrm{wt} \%)$ & $(\mathrm{min})$ & \\
\hline HMPE-1 & 99.50 & 0.50 & 2.1 & 360 & 3.7 \\
HMPE-2 & 99.00 & 1.00 & 3.9 & 370 & 3.7 \\
HMPE-3 & 99.00 & 1.00 & 3.0 & 390 & 4.8 \\
HMPE-4 & 99.00 & 1.00 & 2.5 & 300 & 6.0 \\
HMPE-5 & 98.50 & 1.50 & 5.7 & 410 & 3.7 \\
\hline
\end{tabular}

\section{S3. CMC and $N_{\text {agg }}$ of SDS in a $6 \%$ AA Aqueous Solution at $60{ }^{\circ} \mathrm{C}$}

Due to the addition of AA, the whole system is acidic during the micellar polymerization reaction. In addition, the reaction proceeded at $60{ }^{\circ} \mathrm{C}$. Previous studies ${ }^{1,2}$ reported that $\mathrm{pH}$ and temperature have a strong influence on the critical micelle concentration (CMC) and $N_{\text {agg }}$ of SDS. Thus, it is necessary to determine the CMC and $N_{\text {agg }}$ of SDS in the reaction environment.

The CMC of SDS was measured by a fluorescent probe method using pyrene as a probe molecule. First, $20 \mu \mathrm{L}$ of a $2.5 \times 10^{-3} \mathrm{M}$ pyrene solution in methanol was added to a $10 \mathrm{~mL}$ brown glass volumetric flask, and then the methanol was dried with nitrogen gas. After being completely dried, 6\% acrylic acid (AA) aqueous solutions with different SDS concentrations were added to 
the bottle, and the solutions were ultrasonicated for $5 \mathrm{~min}$ before fluorescence emission measurements at $60{ }^{\circ} \mathrm{C}$. The results show that the CMC was approximately $7 \mathrm{mM}$ (the CMC was defined as the concentration when pyrene exhibited an apparent decrease in the $I_{1} / I_{3}$ ratio with increasing SDS concentration, indicating that the aggregation of the SDS molecules occurred (Figure S1A). Furthermore, the spectra are shown in Figure S1B.
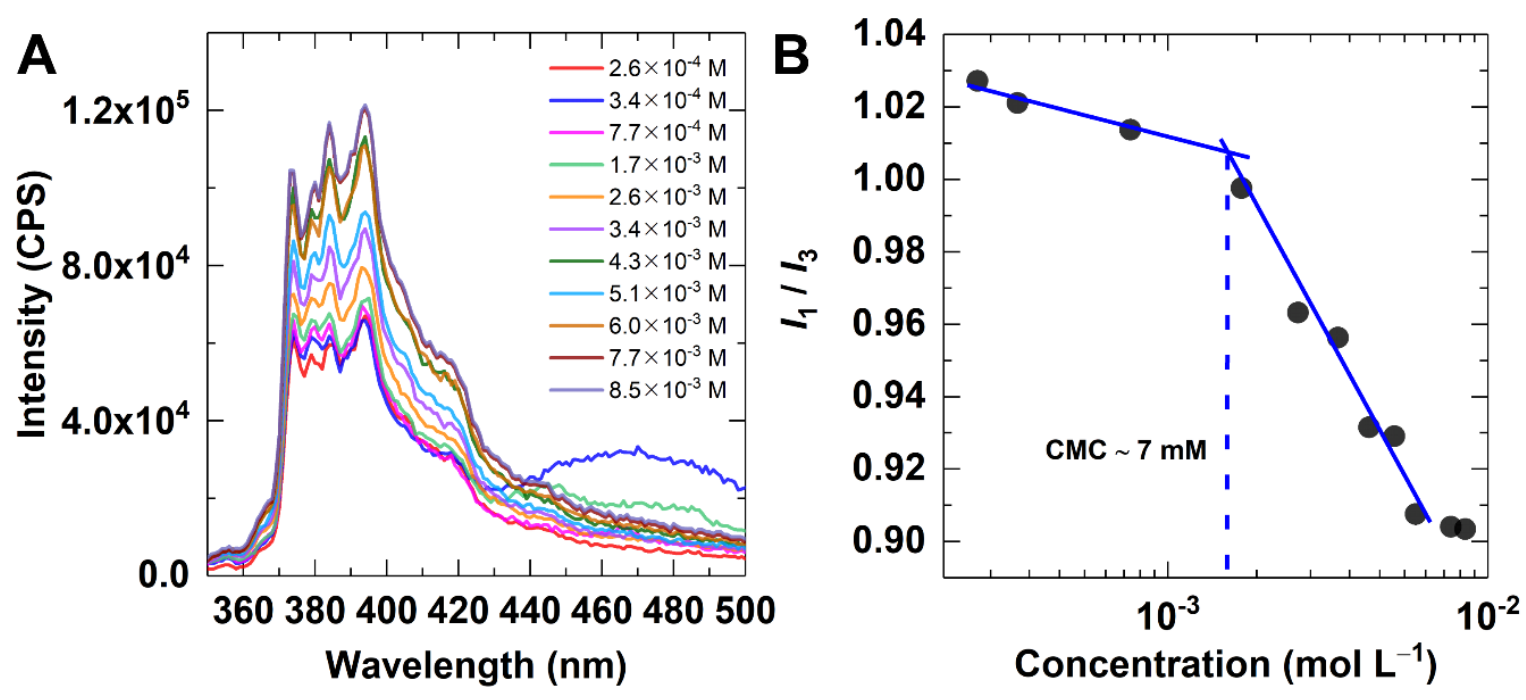

Figure S1. (A) Fluorescence emission spectra of pyrene in 6\% AA aqueous solutions with different SDS concentrations at $60{ }^{\circ} \mathrm{C}$. (B) Variation in the $I_{1} / I_{3}$ value with SDS concentration in a $6 \%$ AA aqueous solution at $60^{\circ} \mathrm{C}$.

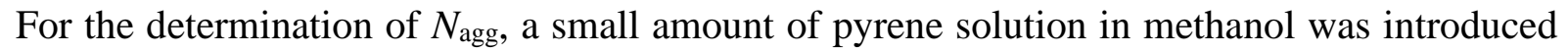
into an Erlenmeyer flask. Then, methanol dried under nitrogen gas and $3 \mathrm{wt} \%$ SDS in 6\% AA aqueous solution were introduced into the flask. This solution, S1, was stirred for $24 \mathrm{~h}$ at room temperature. The final pyrene concentration was approximately $5 \times 10^{-6} \mathrm{M}$. Then, solution S1 was added to flasks without and with dodecylpyridinium chloride (quencher, the quencher was in methanol, and the methanol was dried with nitrogen before being introduced to S1). The final dodecylpyridinium chloride concentrations in the two flasks were 0 and $0.0016 \mathrm{M}$, respectively.

The $I$ obtained from the pyrene fluorescence spectrum at $373 \mathrm{~nm}$ was used to estimate the micelle concentration from the steady-state fluorescence quenching experiments performed in the 
presence of a fluorescence quencher, Q. Dodecylpyridinium chloride is generally used for studying SDS micelles. The molar concentration of the micelles $\left[\mathrm{M}_{\mathrm{i}}\right]$ was obtained from the fluorescence intensity decrease of the probe (pyrene) as a function of [Q], and the relation is ${ }^{3,4}$

$I=I_{0} \exp \left(-[Q] /\left[M_{\mathrm{i}}\right]\right)$

where $I_{0}$ is the fluorescence intensity in the absence of the quencher, and $\left[\mathrm{M}_{\mathrm{i}}\right]$ is the concentration of micelles.

From $\left[\mathrm{M}_{\mathrm{i}}\right]$, the aggregation number of SDS in solution $N_{\text {agg }}$ can be calculated as $N_{\mathrm{agg}}=([S D S]-\mathrm{cmc}) /\left[M_{\mathrm{i}}\right]$

where $[S D S]$ is the known concentration of SDS and its CMC is $7 \mathrm{mM}$.

The $I_{0}$ and $I$ for $3 \mathrm{wt} \%$ SDS with 0 and $0.0016 \mathrm{M}$ quencher in 6\% AA water solution are displayed in Figure S2, where $I_{0}=94827$ and $I=35315$. From eq S1, $\left[M_{\mathrm{i}}\right]$ is $0.0016 \mathrm{M}$. Then, $\left[M_{\mathrm{i}}\right]=0.0016 \mathrm{M}, \mathrm{CMC}=7 \times 10^{-3} \mathrm{M}$ and $[S D S]=0.104 \mathrm{M}(3 \mathrm{wt} \%)$ were used in eq S2 to calculate $N_{\text {agg, }}$ which was found to be 60 .

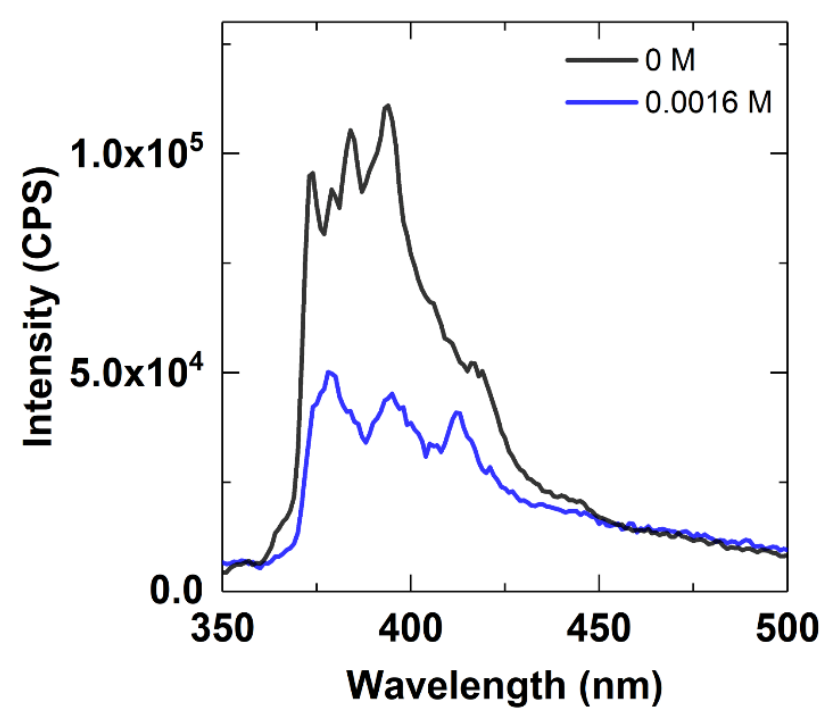

Figure S2. Fluorescence emission spectra of pyrene in a 6\% AA water solution with 0 and 0.0016 $\mathrm{M}$ quencher at $60^{\circ} \mathrm{C}(\mathrm{SDS}=3 \mathrm{wt} \%)$.

\section{S4. Effect of Protonation Degree on HMPE-5}


The changes in $I_{1} / I_{3}$ and zero-shear viscosity as a function of the protonation degree $\delta$ of 0.2 wt\% HMPE-5 in water are displayed in Figure S3. As the protonation degree increases, the $I_{1} / I_{3}$ and $\mathrm{pH}$ values decrease, indicating that the hydrophobic microenvironment is strengthened by protonation of the sodium acrylate unite. In contrast, the zero-shear viscosity first increases and ultimately decreases as the protonation degree increases. This behavior can be attributed to the fact that at the beginning, the charge on the polymer backbone was shielded by protonation, thus enhancing the hydrophobic effect, which increased the viscosity. Upon protonation, the screening of the electrostatic repulsive force of the negative charges along the chain backbone causes the chain to curl, resulting in a decrease in viscosity. As the zero-shear viscosity at $\delta=0.44(\mathrm{pH}=4.9)$ is the largest, all the samples in this study were protonated at $\delta=0.44$ for fluorescence spectroscopy and rheological measurements.

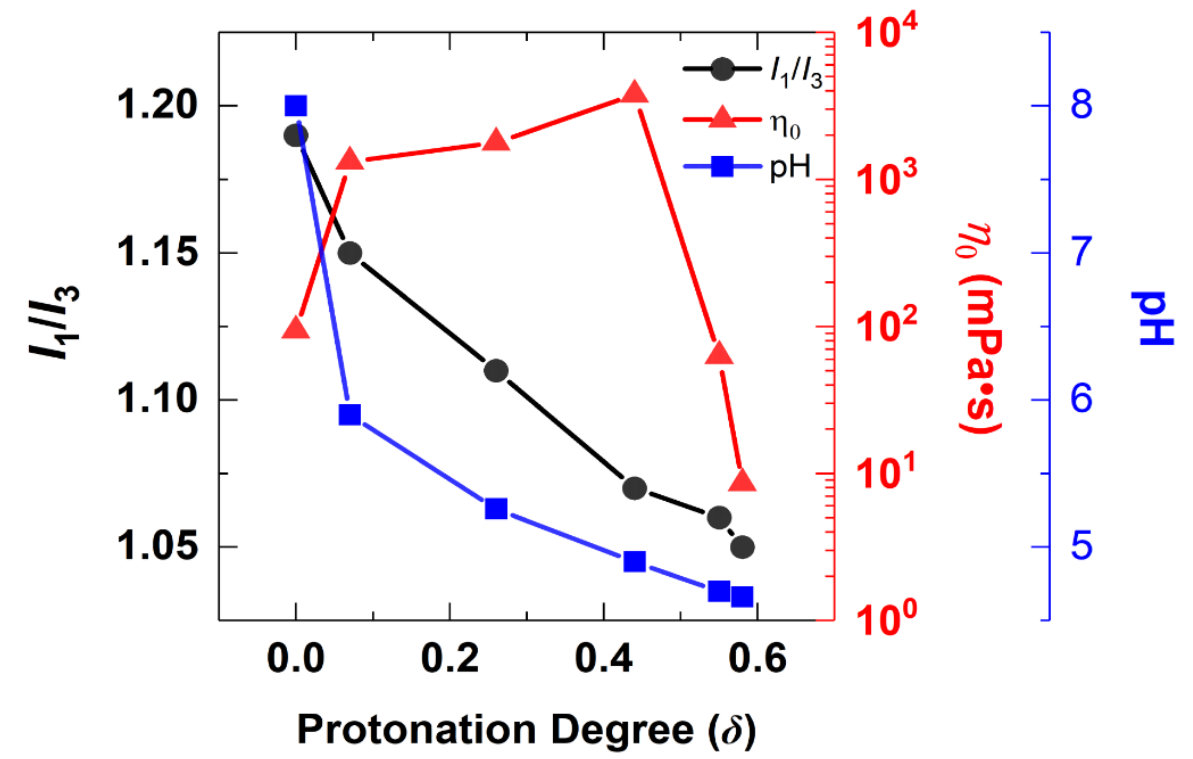

Figure S3. The changes in $I_{1} / I_{3}$, zero-shear viscosity and $\mathrm{pH}$ as a function of the protonation degree $\delta$ in water (sample is HMPE-5, and $C_{\mathrm{p}}=0.2 \mathrm{wt} \%$ ).

\section{S5. Polymerization Kinetics and Compositional Heterogeneity}

In the process of micellar polymerization, samples (HMPE-1, 2, 3, 4 and 5) were removed at different reaction times during 8 hours and weighed. Then, the samples were dissolved in $0.4 \%$ 
hydroquinone to stop the reaction and precipitated by slowly pouring them into a constantly stirred volume of 6-fold excess ethyl acetate. After filtration, the polymer was obtained by freeze-drying for 2 days. The AA units were not ionized. The monomer conversion was determined based on the following equation:

$C_{\mathrm{p}}=p /\left(M_{\mathrm{e}} \times C_{\mathrm{m}}\right) \times 100 \%$

where $\mathrm{p}$ is the mass of the product, $M_{\mathrm{e}}$ is the mass of the sample, and $C_{\mathrm{m}}$ is the monomer concentration.

The monomer conversion as a function of reaction time is displayed in Figure S4A. The conversion increases as the reaction time increases. The conversion of HMPE-1, -2 and -5 with the same $N_{\mathrm{H}}=3.7$ showed a similar trend, while HMPE-3 with $N_{\mathrm{H}}=4.8$ and HMPE-4 with $N_{\mathrm{H}}=6.0$ showed slower conversion than HMPE-1, -2 and -5 as the reaction time increased. This phenomenon is attributed to the higher $N_{\mathrm{H}}$ with more hydrophobes in each micelle increasing the steric hindrance during the polymerization process. Furthermore, the hydrophobe content as a function of time is also displayed in Figure S4B. Clearly, the hydrophobe content shifts as the reaction time increases. For this reason, different reaction times were used for different samples of the HMPEs to ensure that HMPE-1, -2 and -5 incorporated different hydrophobe contents and that HMPE-2, 3 and 4 contained the same hydrophobe content. The reaction times are listed in Table S1. 

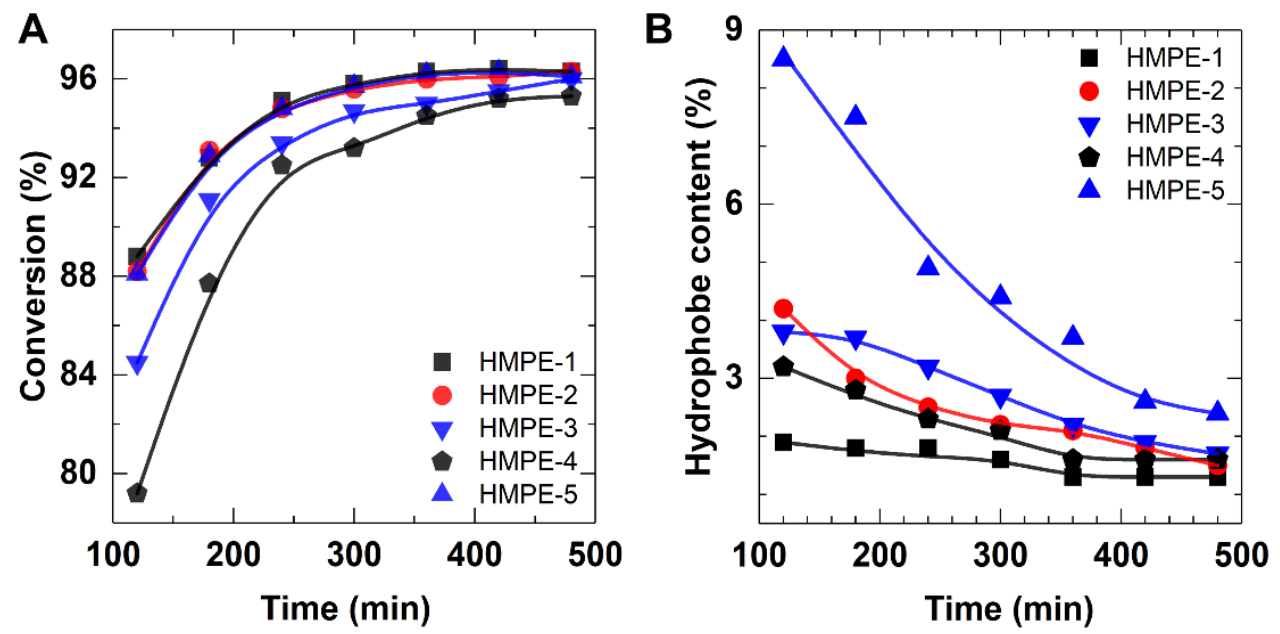

Figure S4. (A) Monomer conversion as a function of time for various polymers. (B) Incorporation of SMA as a function of the reaction time for various polymers. 
S6. Zimm Plots and Molecular Weight
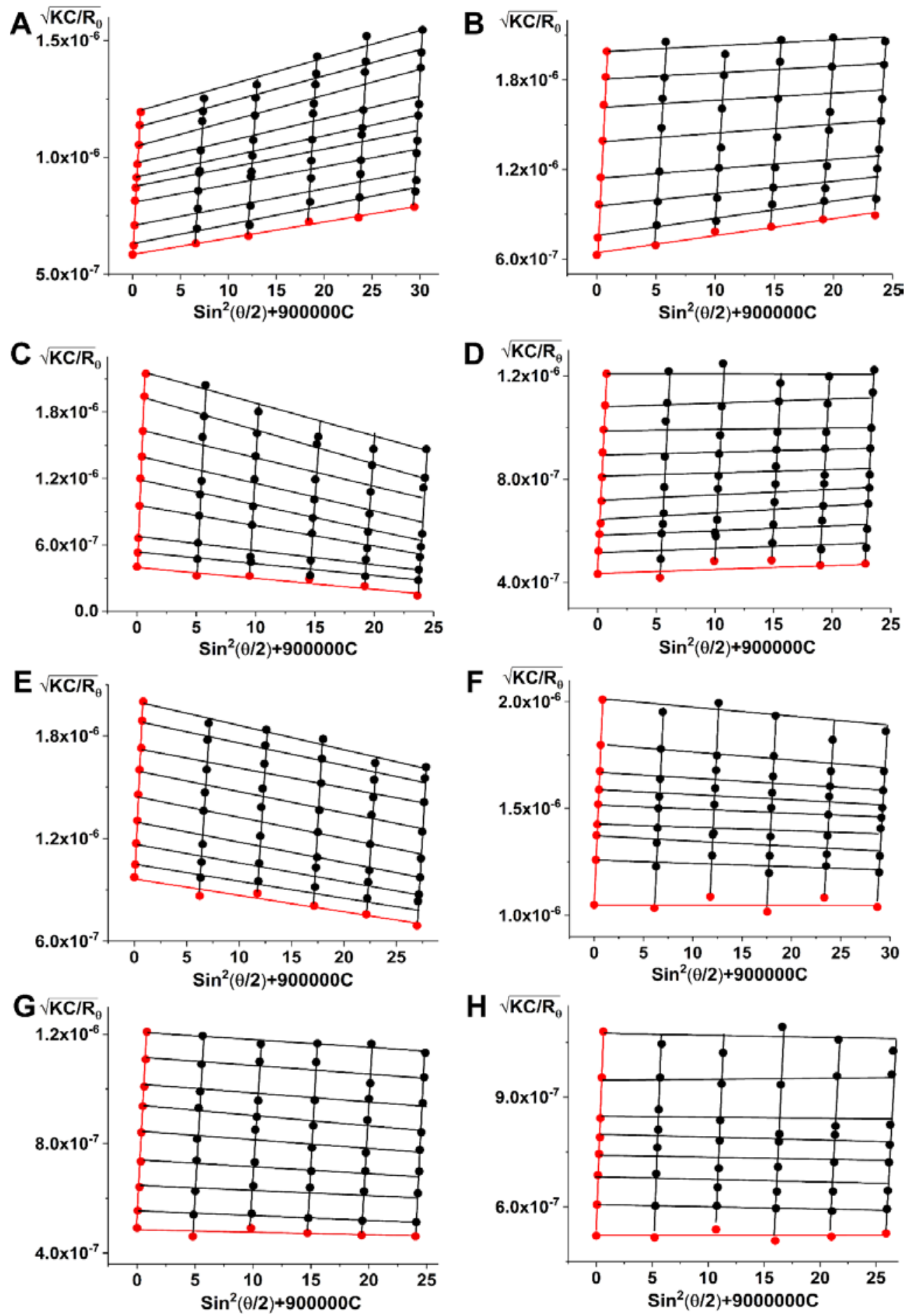

Figure S5. Zimm plots of (A) HMPE-2; (B) hHMPE-2; (C) HMPE-3; (D) hHMPE-3; (E) HMPE4; (F) hHMPE-4; (G) HMPE-5; and (H) hHMPE-5. 


\section{S7. Calculation of Theoretical Molecular Weight of Polymers}

The molecular weight of the hydrolyzed HMPEs (hHMPEs) is calculated by the following equation:

$M_{\mathrm{w}, \mathrm{T}}=\frac{\mathrm{m}_{\mathrm{A}} \times \mathrm{m}+\mathrm{m}_{\mathrm{CH}_{3}} \times \mathrm{n}}{\mathrm{m}_{\mathrm{A}} \times \mathrm{m}+\mathrm{m}_{\mathrm{S}} \times \mathrm{n}} \times M_{\mathrm{w}}==\frac{94 \mathrm{~m}+15 \mathrm{n}}{94 \mathrm{~m}+338 \mathrm{n}} \times M_{\mathrm{w}}$

where $m_{A}, m_{C H 3}$, and $m_{S}$ are the molecular masses of sodium acrylate, the $-\mathrm{CH}_{3}$ pendant group in the polymer backbone and SMA, respectively; $\mathrm{m}$ and $\mathrm{n}$ denote the sodium acrylate and hydrophobe contents, respectively, which are determined from the ${ }^{1} \mathrm{H}$ NMR spectra; and $M_{\mathrm{w}}$ refers to the molecular weights of HMPE-1, HMPE-2, HMPE-3, HMPE-4 and HMPE-5, which are determined by static light scattering (SLS). 


\section{S8. Fluorescence Spectra}
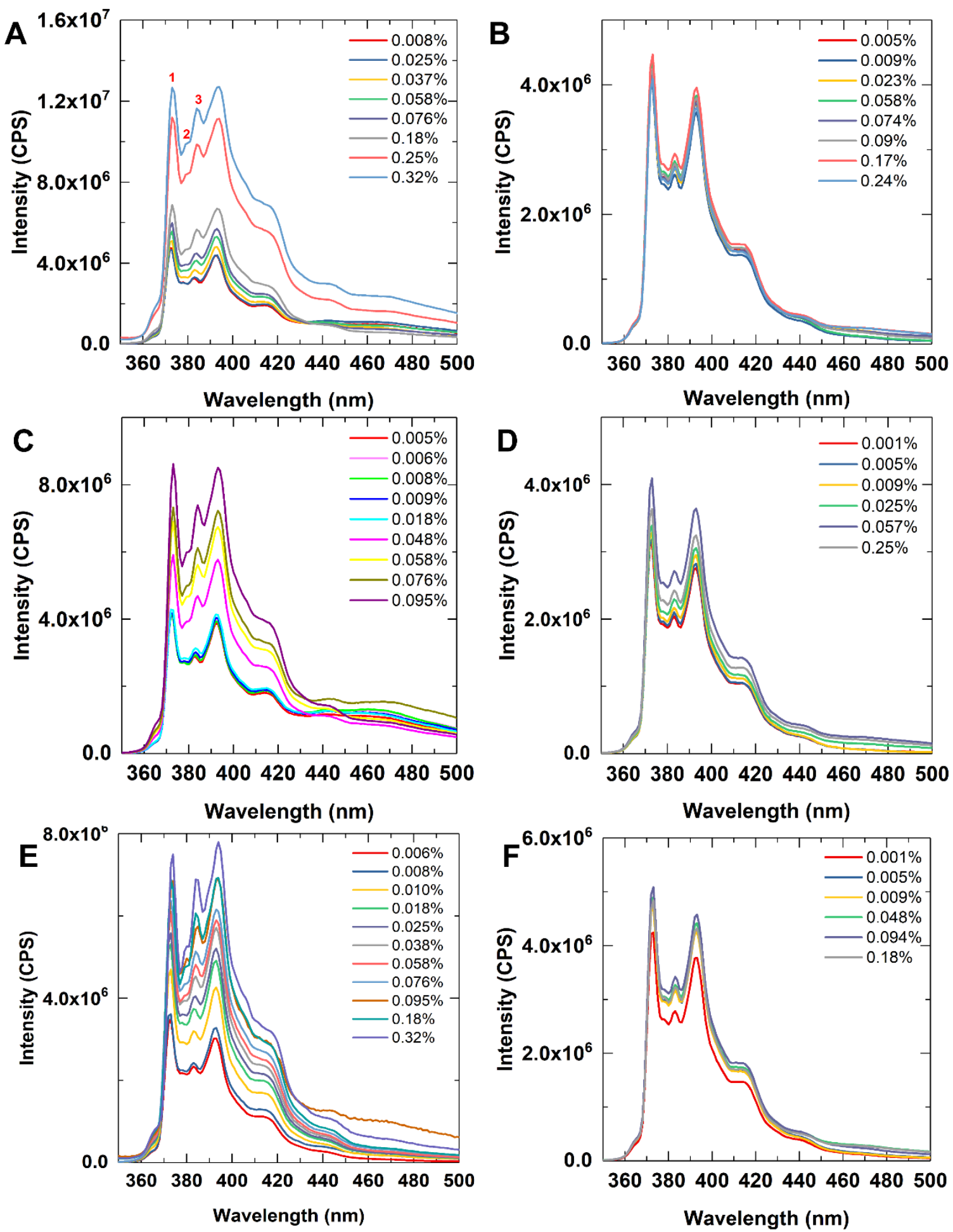

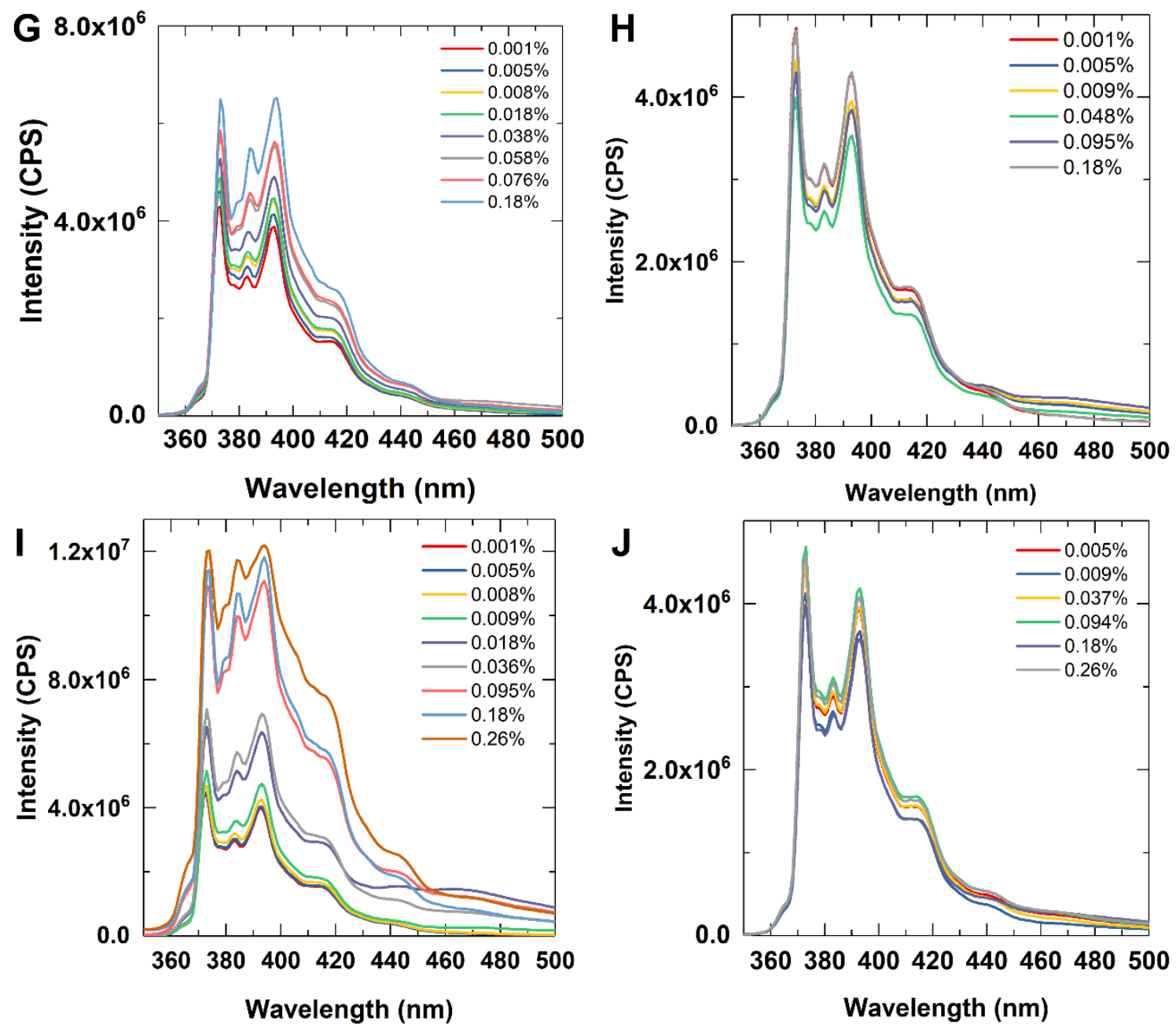

Figure S6. Fluorescence spectra of (A) HMPE-1; (B) hHMPE-1; (C) HMPE-2; (D) hHMPE-2; (E) HMPE-3; (F) hHMPE-3; (G) HMPE-4; (H) hHMPE-4; (I) HMPE-5; and (J) hHMPE-5 in deionized water. 

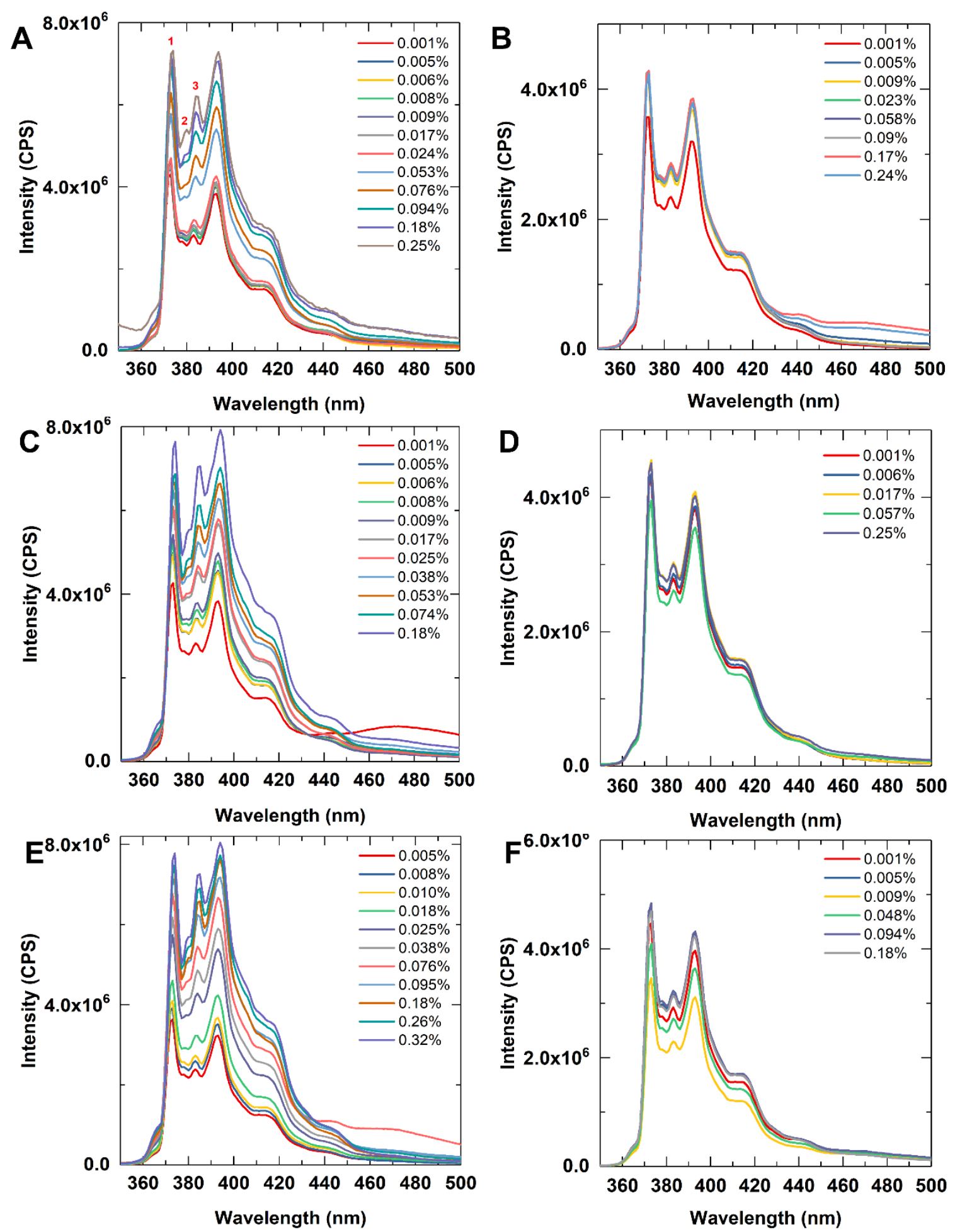

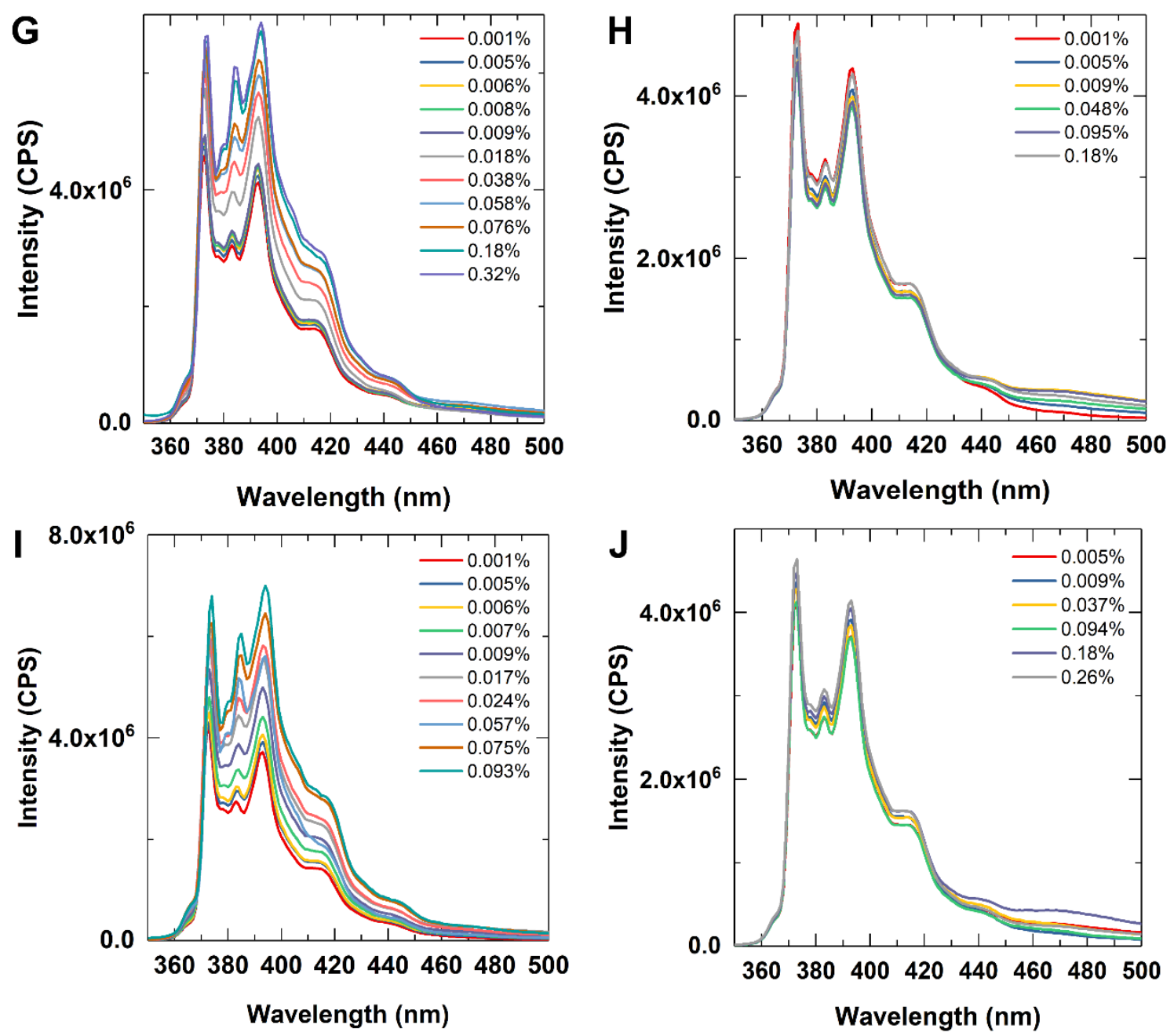

Figure S7. Fluorescence spectrum of (A) HMPE-1; (B) h HMPE-1; (C) HMPE-2; (D) hHMPE-2; (E) HMPE-3; (F) hHMPE-3; (G) HMPE-4; (H) hHMPE-4; (I) HMPE-5; and (J) hHMPE-5 in a $0.02 \mathrm{M} \mathrm{NaCl}$ solution.

\section{S9. Steady Rheology in Deionized Water}

The flow curves of the HMPEs and hHMPEs at different concentrations in deionized water are presented in Figure S9. One can observe that the shear viscosity increases with increasing polymer concentration. At lower polymer concentrations, both the HMPEs and hHMPEs behave as a Newtonian fluid, with a plateau region of constant viscosity. At higher concentrations, the hHMPE solutions exhibit a Newtonian region at beginning followed by shear-thinning behavior, which is 
attributed to the increase in the alignment of the polymer molecules in the flow direction by shear. ${ }^{5}$ In contrast, the solutions of the HMPEs at high concentration behave as a pseudoplastic fluid: after a Newtonian plateau at low shear rates, the solutions show pronounced shear thickening followed by shear thinning. Similar behaviors were reported previously for an HMPE prepared by micellar copolymerization. ${ }^{6}$ However, this behavior was not always obtained from copolymers prepared by micellar polymerization. ${ }^{7}$ The size of the hydrophobic blocks is more important to the associative properties than the number of hydrophobic blocks. ${ }^{8}$ 

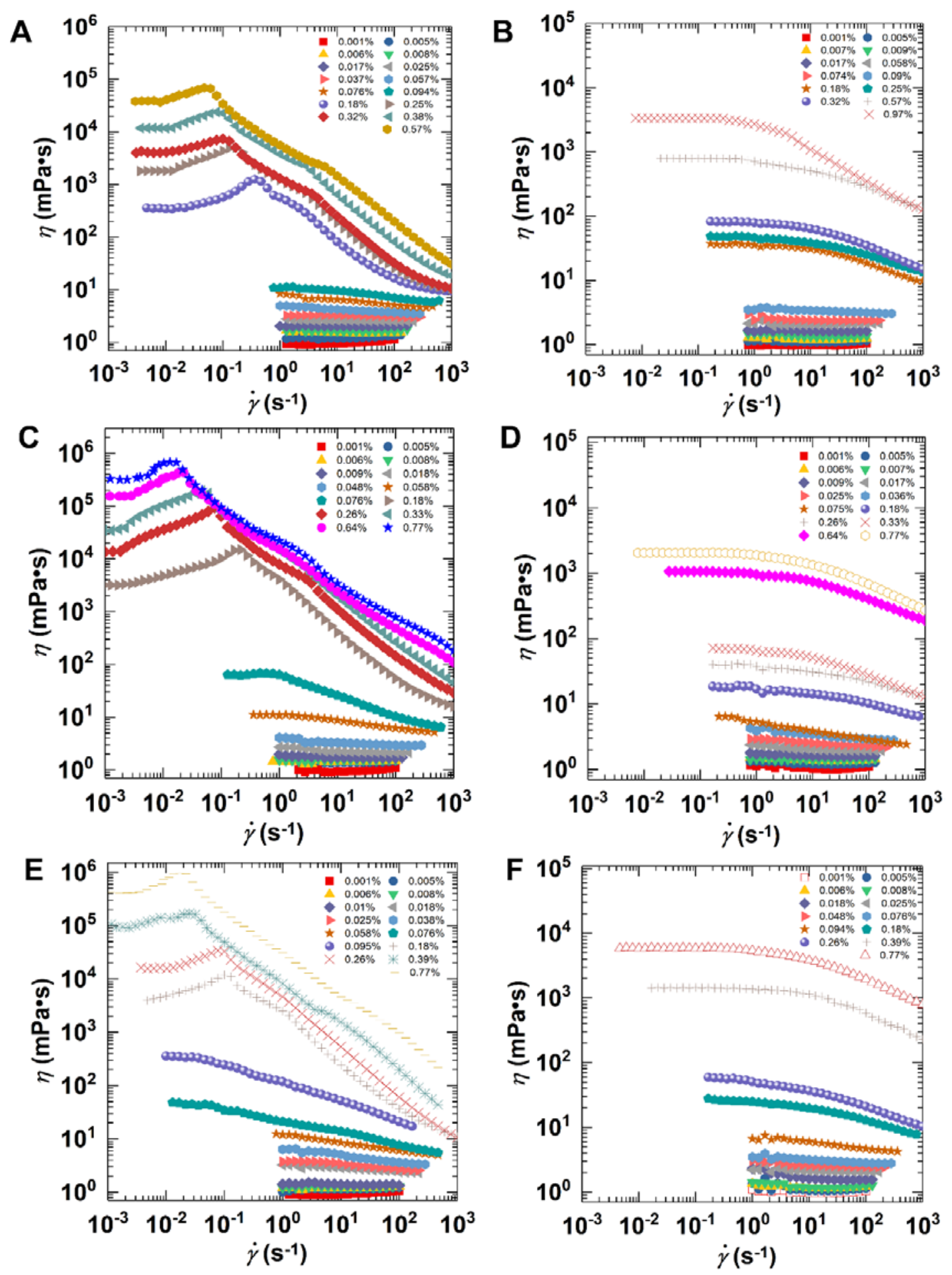

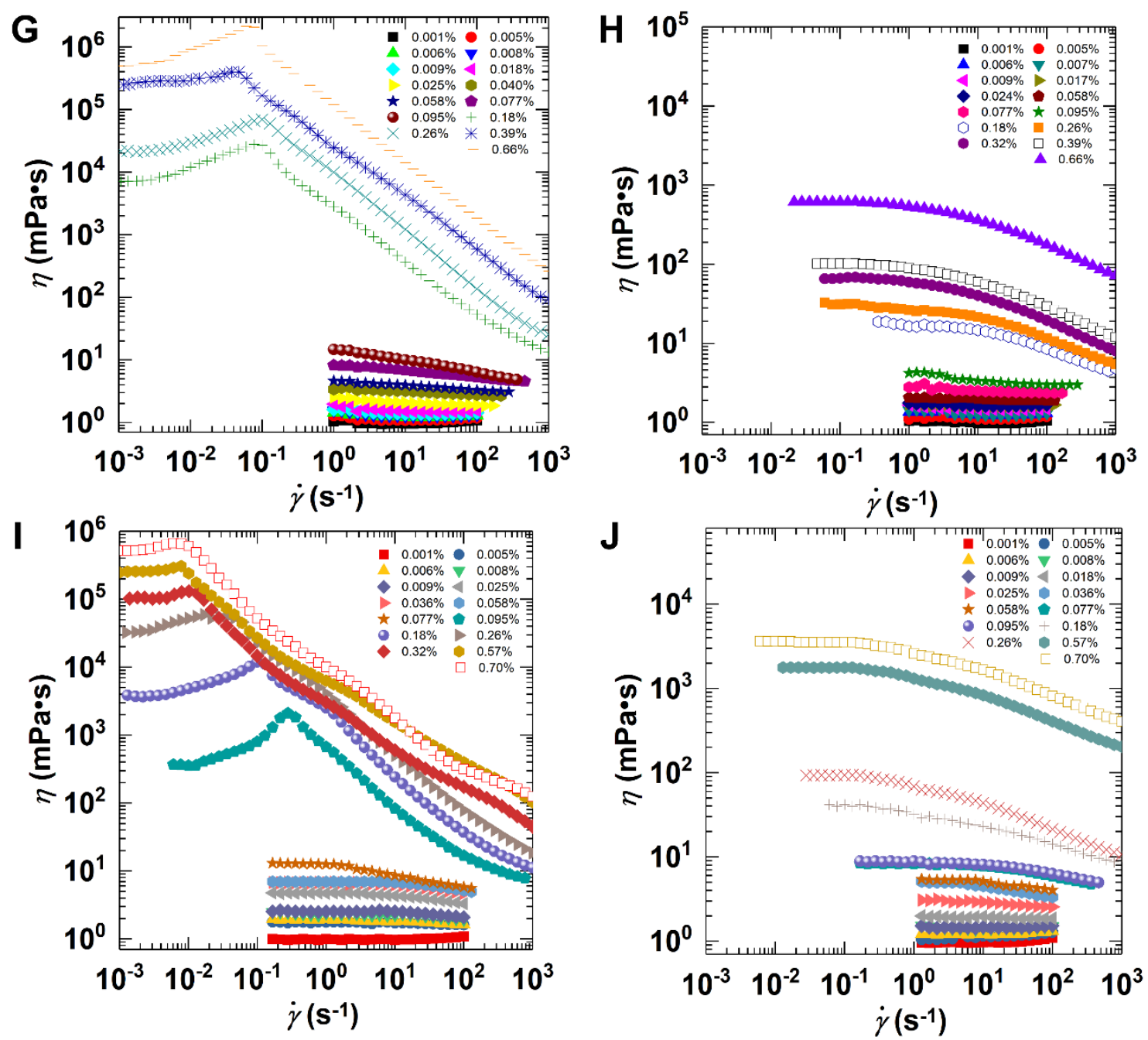

Figure S8. Viscosity as a function of shear rate for HMPEs and hHMPEs in deionized water at different concentrations: (A) HMPE-1; (B) hHMPE-1; (C) HMPE-2; (D) hHMPE-2; (E) HMPE3; (F) hHMPE-3; (G) HMPE-4; (H) hHMPE-4; (I) HMPE-5; and (J) hHMPE-5.

S10. Zero-Shear-Rate Viscosity of Aqueous HMPEs and hHMPEs Solutions in the Dilute and Semidilute Unentangled Regimes 

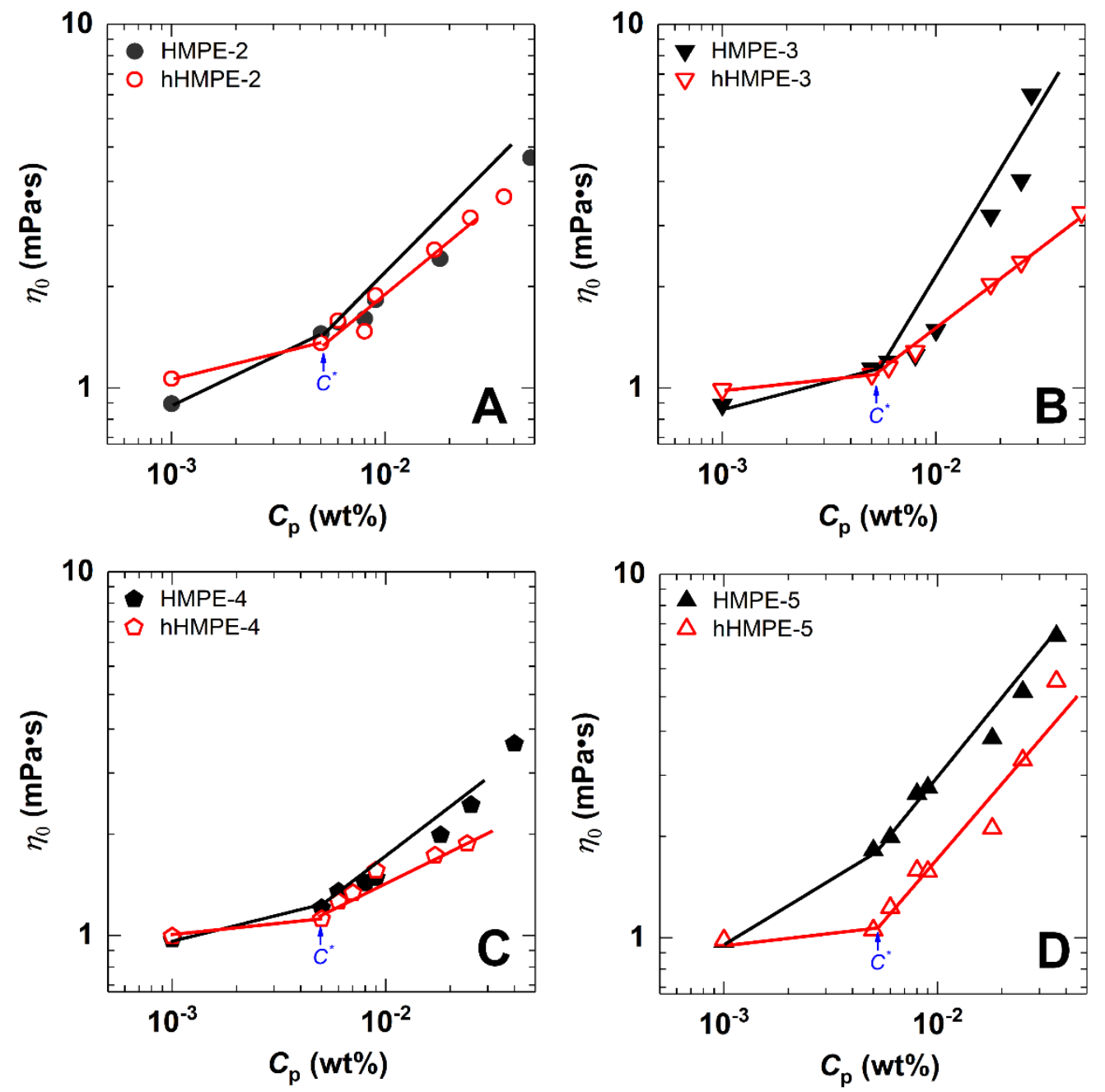

Figure S9. Comparison of the variation of the zero shear rate viscosity of the polymers in pure water as a function of the copolymer concentration for HMPEs and the corresponding hydrolyzed polymers in the dilute and semidilute unentangled regimes: (A) HMPE-2 and hHMPE-2; (B) HMPE-3 and hHMPE-3; (C) HMPE-4 and hHMPE-4; and (D) HMPE-5 and hHMPE-5.

\section{S11. Calculation of the Concentration of Hydrophobic Groups in Solution}

In the rheological study, the polymers were protonated by titration with a hydrochloric acid solution. Therefore, charged AA units and neutral AA units exist in the polymer backbone. Assume that there are $n_{\mathrm{A}}$ mol of charged AA units, $\mathrm{n}_{\mathrm{AA}}$ mol of neutral AA units and $\mathrm{n}_{\mathrm{s}}$ mol of SMA units 
in $1 \mathrm{~L}$ aqueous solution. Therefore, the molar concentration of hydrophobic group in solution $C_{\mathrm{H}}$ can be calculated by using the following equation:

$C_{\mathrm{H}}=\frac{\mathrm{C}_{\mathrm{p}} / 100 \times 1000 \times[\mathrm{H}]}{\left[\mathrm{m}_{\mathrm{A}} \times(1-\delta)+\mathrm{m}_{\mathrm{AA}} \times \delta\right] \times(1-[\mathrm{H}])+\mathrm{m}_{\mathrm{S}} \times[\mathrm{H}]}$

where $\mathrm{m}_{\mathrm{A}}$ is the molecular mass of sodium acrylate, $94 \mathrm{~g} / \mathrm{mol}$; $\mathrm{m}_{\mathrm{AA}}$ is the molecular mass of $\mathrm{AA}$, $72 \mathrm{~g} / \mathrm{mol} ; \delta$ is the protonated degree of HMPEs which is equal to 0.44 in this study; $\mathrm{ms}_{\mathrm{s}}$ is the molecular mass of SMA, $328 \mathrm{~g} / \mathrm{mol}$, respectively.

\section{S12. Neutral Polymer System}

In our study, the range of concentrations of the polymer solutions was $0.001 \mathrm{wt} \% \leq C_{\mathrm{p}}<1 \mathrm{wt} \%$. As the portions of hydrophobic units in the HMPEs and methyl groups in the hHMPEs are as low as $2.7 \%$, the molecular mass of those groups is neglected, and then the molar concentration of the monomer units $C_{\mathrm{m}}$ and $\mathrm{A}$ can be calculated using the following equation:

$C_{\mathrm{m}}=C_{\mathrm{p}} \times 1000 /[72 \times 0.44+94 \times(1-0.44)]$

In this section, the protonation of the carboxylate groups in the polymer is random, and the titration degree is 0.44 . Then, there are two extremes in the distribution of charged units: all the charged units are singly distributed in the polymer backbone, where $\mathrm{A}=0.44 / 0.56=0.78$, and all the charged unites are together in the middle of the backbone, where $A=22$. However, not all the charge distributions are in one of these two situations. Here, we assume that five charged units are distributed together in the polymer backbone; thus, $\mathrm{A}=0.44 /(0.56 / 5)=3.92$.. Therefore, the range of $C_{\mathrm{m}} / 2 \mathrm{~A}$ is $2.0 \times 10^{-5} \mathrm{~mol} \cdot \mathrm{L}^{-1} \leq C_{\mathrm{m}} / 2 \mathrm{~A}<0.015 \mathrm{~mol} \cdot \mathrm{L}^{-1}$. The highest value of $C_{\mathrm{m}} / 2 \mathrm{~A}$ is lower than $C_{\mathrm{s}}(0.02 \mathrm{M})$. However, in our case, most of the polymer concentrations are far lower than $1 \mathrm{wt} \%$. Thus, the addition of $0.02 \mathrm{M}$ salt is enough to screen the ionic strength.

\section{S13. Steady Rheology in a $0.02 \mathrm{M} \mathrm{NaCl}$ Solution}

Figure S9 presents an illustration of the steady-state flow response of HMPEs and hHMPEs at different concentrations in a $0.02 \mathrm{M} \mathrm{NaCl}$ aqueous solution. From Figure S9, one can observe the 
same trends as those in Figure S8; however, the viscosity in $0.02 \mathrm{M} \mathrm{NaCl}$ is lower than that in deionized water from Figure S8 because of the screening of charges in the polymer backbone by salt.
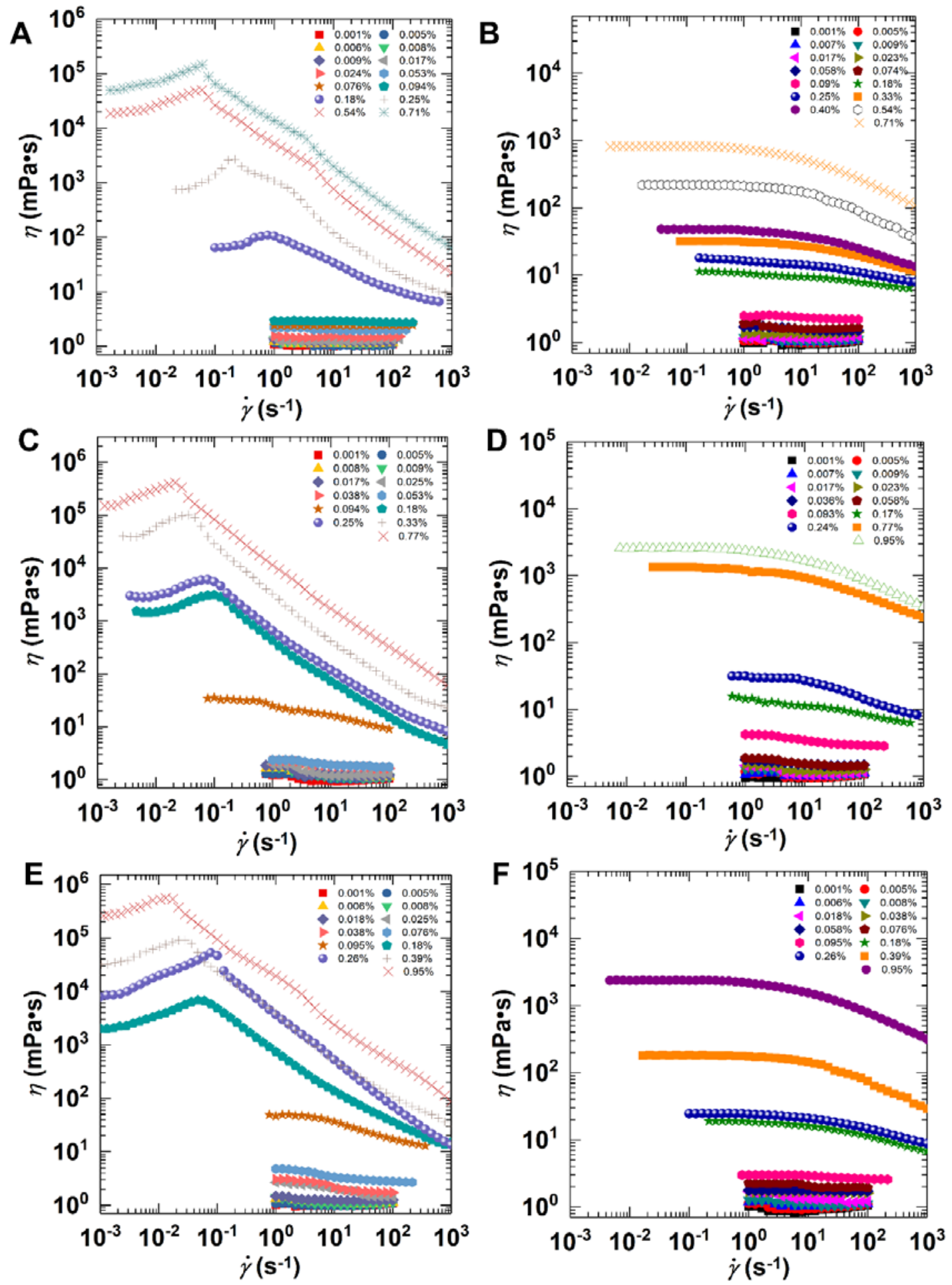

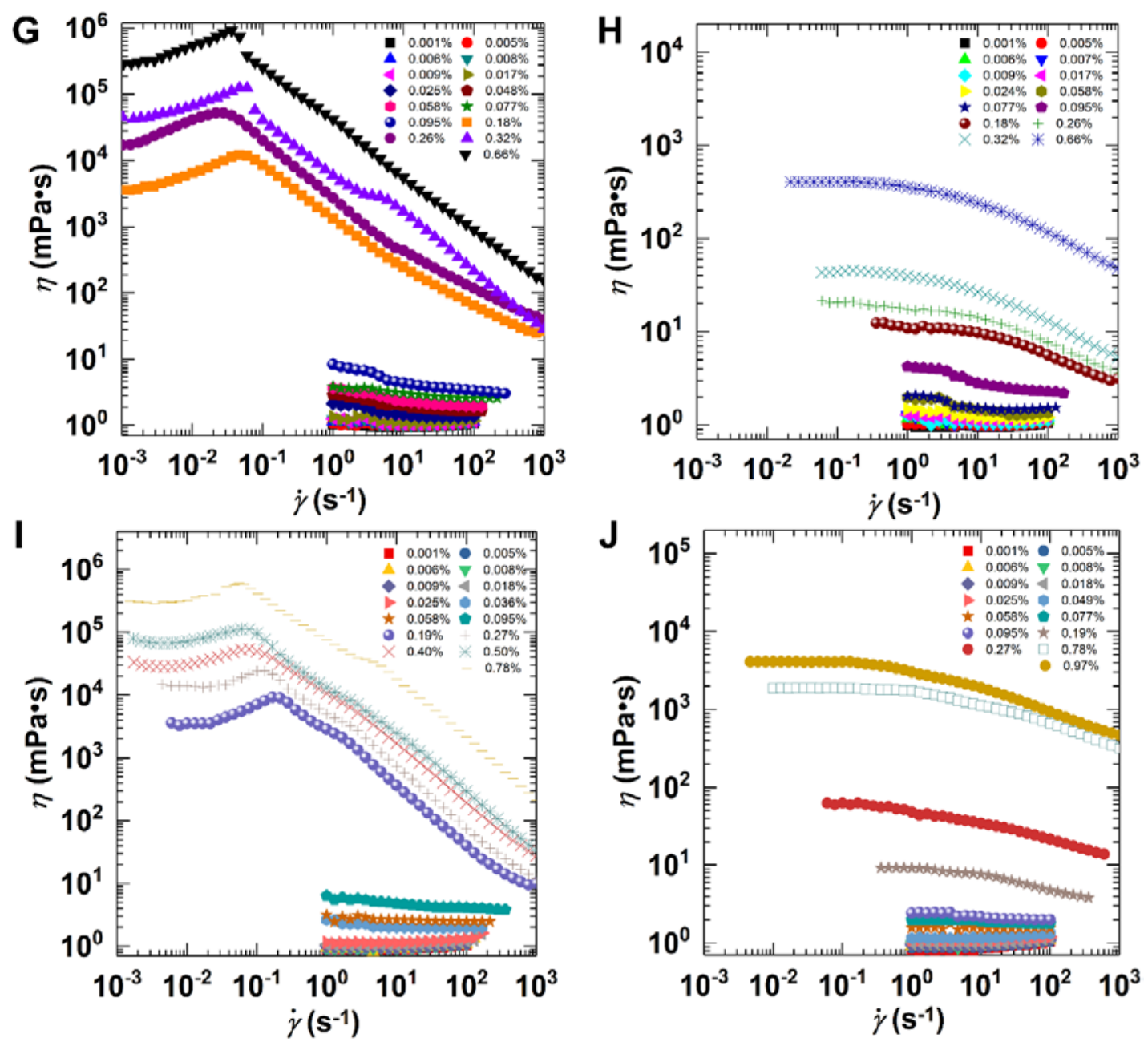

Figure S10. Viscosity as a function of shear rate for HMPEs and hHMPEs in $0.02 \mathrm{M} \mathrm{NaCl}$ at different concentrations: (A) HMPE-1; (B) hHMPE-1; (C) HMPE-2; (D) hHMPE-2; (E) HMPE3; (F) hHMPE-3; (G) HMPE-4; (H) hHMPE-4; (I) HMPE-5; and (J) hHMPE-5.

S14. Zero-Shear-Rate Viscosity for HMPEs and hHMPEs in $0.02 \mathrm{M}$ NaCl Solution in the Dilute and Semidilute Unentangled Regimes 

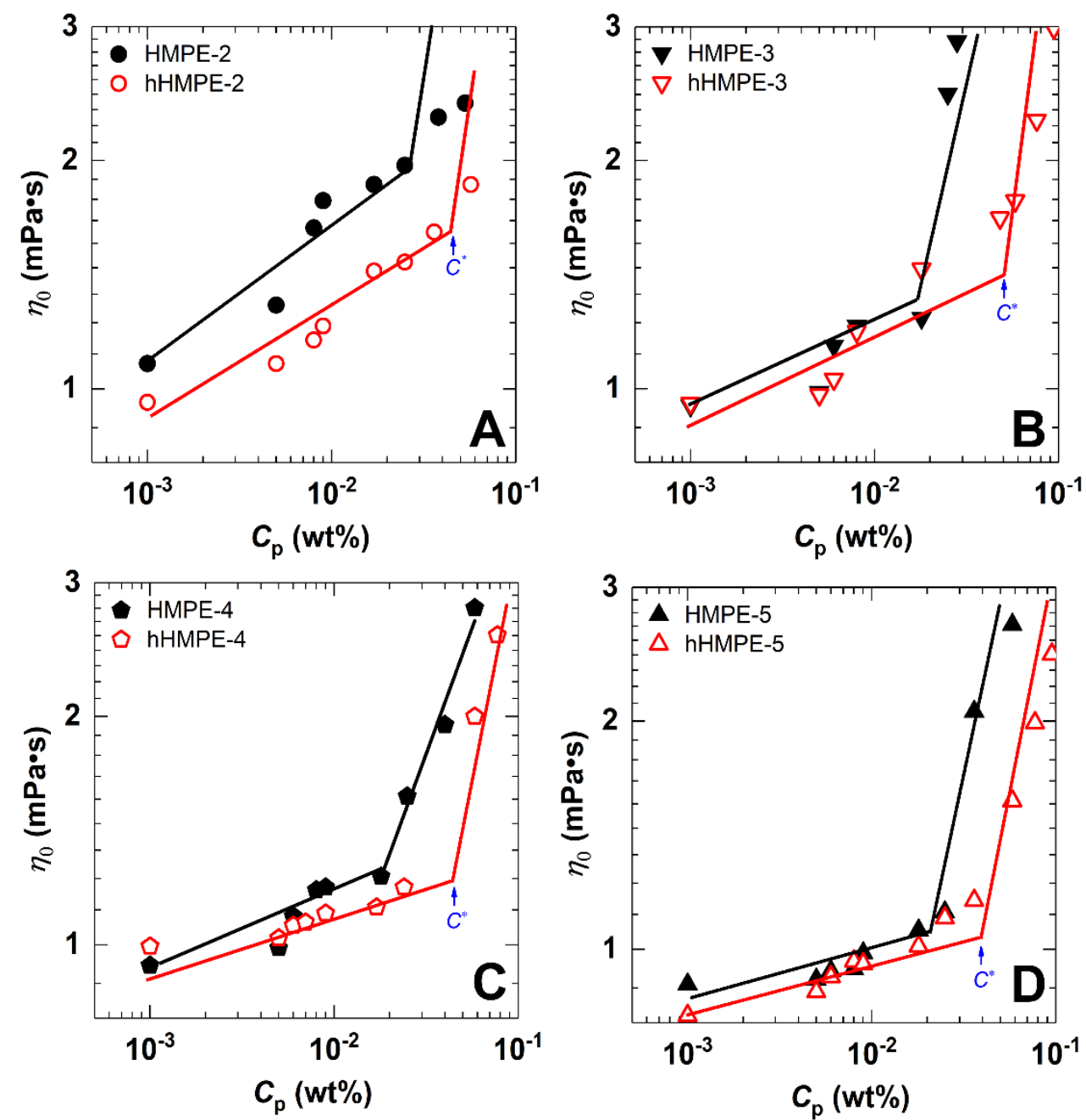

Figure S11. Comparison of the variation in the zero shear rate viscosity of the polymers in 0.02 $\mathrm{M} \mathrm{NaCl}$ as a function of the copolymer concentration for HMPEs and the corresponding hydrolyzed polymers in the dilute and semidilute unentangled regimes: (A) HMPE-2 and hHMPE2; (B) HMPE-3 and hHMPE-3; (C) HMPE-4 and hHMPE-4; and (D) HMPE-5 and hHMPE-5. 


\section{References}

(1) Rahman, A.; Brown, C. Effect of pH on the Critical Micelle Concentration of Sodium Dodecyl Sulphate. J. Appl. Polym. Sci. 1983, 28, 1331-1334.

(2) Matijević, E.; Pethica, B. A. The Heats of Micelle Formation of Sodium Dodecyl Sulphate. Trans. Faraday Soc. 1958, 54, 587-592.

(3) Turro, N. J.; Yekta, A. Luminescent Probes for Detergent Solutions. A Simple Procedure for Determination of the Mean Aggregation Number of Micelles. J. Am. Chem. Soc. 1978, 100, 5951-5952.

(4) Bastiat, G.; Grassl, B.; Khoukh, A.; François, J. Study of Sodium Dodecyl Sulfate-Poly (Propylene Oxide) Methacrylate Mixed Micelles. Langmuir 2004, 20, 5759-5769.

(5) Castillo-Tejas, J.; Castrejón-González, O.; Carro, S.; González-Coronel, V.; Alvarado, J.; Manero, O. Associative Polymers. Part III: Shear Rheology from Molecular Dynamics. Colloids Surf., A 2016, 491, 37-49.

(6) Biggs, S.; Selb, J.; Candau, F. Copolymers of Acrylamide/N-Alkylacrylamide in Aqueous Solution: The Effects of Hydrolysis on Hydrophobic Interactions. Polymer 1993, 34, 580-591.

(7) Lacík, I.; Selb, J.; Candau, F. Compositional Heterogeneity Effects in Hydrophobically Associating Water-Soluble Polymers Prepared by Micellar Copolymerization. Polymer 1995, 36, 3197-3211.

(8) Volpert, E.; Selb, J.; Candau, F. Associating Behaviour of Polyacrylamides Hydrophobically Modified with Dihexylacrylamide. Polymer 1998, 39, 1025-1033. 